\title{
Incidence and risk of developing contrast-induced acute kidney injury following intravascular contrast administration in elderly patients
}

This article was published in the following Dove Press journal:

Clinical Interventions in Aging

31 December 2013

Number of times this article has been viewed

Wei Song

Tuo Zhang

Jun $\mathrm{Pu}$

Linghong Shen

Ben $\mathrm{He}$

Department of Cardiology,

Ren Ji Hospital, School of Medicine,

Shanghai Jiao Tong University,

Shanghai, People's Republic of China
Background: The purpose of this meta-analysis was to evaluate the epidemiology of contrast-induced acute kidney injury (CI-AKI) in the elderly.

Methods: A literature review was undertaken to determine the incidence of CI-AKI in individuals receiving intravascular contrast medium in the hospital setting.

Results: Twenty-two studies with 186,455 patients were identified. The pooled incidence of CI-AKI was $13.6 \%$ in 67,831 patients older than 65 years of age ( $95 \%$ confidence interval [CI] 10.1-18.2, $\left.I^{2}=0.496\right)$. The pooled odds ratio of CI-AKI in the elderly was $2.55(95 \%$ CI $\left.1.85-3.52, I^{2}=0.34\right)$. The high incidence of CI-AKI in the elderly was consistent across different administration route subgroups (intracoronary contrast medium group, $15.5 \%$ [95\% CI 10.3-22.6]; intravenous contrast medium group, 12.4\% [95\% CI 8.0-18.8]).

Conclusion: Elderly patients are at greater risk for developing CI-AKI.

Keywords: contrast-induced acute kidney injury, angiography, enhanced computed tomography, epidemiology, meta-analysis

\section{Introduction}

Contrast-induced acute kidney injury (CI-AKI) following administration of intravascular contrast media $(\mathrm{CM})$ is currently the third leading cause of hospital-acquired acute kidney injury and occurs in approximately $7 \%$ of unselected patients. ${ }^{1,2}$ Advanced age has been identified as an important risk factor for CI-AKI. ${ }^{3}$ However, limited data exist to determine the actual epidemiology of CI-AKI in elderly patients. To address this issue, we performed a meta-analysis on currently available clinical studies to evaluate the incidence and risk of CI-AKI developing in the elderly.

\section{Materials and methods}

\section{Search strategy}

We performed a MEDLINE literature search from 1966 to June 2013. We derived three comprehensive search themes that were combined by the Boolean operator "AND" (see Supplementary material). For the theme "CI-AKI", the following combinations of medical subject heading terms and text words were used: "contrast induced acute kidney injury", "contrast induced acute renal failure", "contrast nephropathy", “contrast induced nephropathy", "contrast induced nephrotoxicity", "contrast associated nephropathy", "contrast associated nephrotoxicity", and "radiocontrast induced nephropathy". For the theme "elderly", the terms "elderly", "aged", “geriatric”, “older", “senior”, “age group”, “old persons”, “65 years”, “75 years”, and 
"80 years" were used. For the theme "clinical study", we used a previously published search strategy. ${ }^{4}$

\section{Study selection and data extraction}

We included cohort studies and randomized controlled trials that reported the incidence or adjusted odds ratios (ORs) of CI-AKI following intravascular CM administration in elderly patients. An elderly patient was defined as a patient $\geq 65$ years of age. There were no restrictions regarding patient inclusion criteria or administration route of $\mathrm{CM}$. The exclusion criterion was a duplicate report. The studies were reviewed by two independent investigators (WS and TZ) to determine whether the studies met the inclusion criteria. Discrepancies were resolved by consensus between the two review authors when the discrepancy was due to simple oversight by one of these authors. Otherwise, a third author was consulted when the discrepancy was due to a difference in interpretation. Data from included studies were recorded using a standardized form, including the source and design of the studies, inclusion/exclusion criteria, baseline characteristics of participants, and outcomes.

\section{Endpoints, data synthesis, and analysis}

The endpoint was the incidence of CI-AKI, which was defined as an increase in serum creatinine levels of more than $25 \%$ or $0.5 \mathrm{mg} / \mathrm{dL}$ following intravascular $\mathrm{CM}$ administration. ${ }^{2}$ If data for this definition were unavailable, the endpoint of CIAKI reported in the original study was chosen. The overall incidence of CI-AKI in the elderly was estimated with $95 \%$ confidence intervals (CIs) by pooling the reported incidences of CI-AKI from individual studies according to the DerSimonian-Laird random-effect model using MetaAnalyst software (version Beta3.13; Tufts Medical Center, Boston, MA, USA). ${ }^{5}$ The incidence of CI-AKI in prespecified subgroups was also analyzed, including patients aged $\geq 75$ years and $\geq 80$ years of age, with intracoronary CM administration, intra-arterial CM administration other than via the coronary artery, intravenous $\mathrm{CM}$ administration, and administration route other than via coronary artery. Pooled ORs of CI-AKI in elderly patients versus younger patients were estimated according to the inversevariance random-effect model using Review Manager software (Rev-Man Analyses version 5.1.4; Copenhagen, Denmark; The Nordic Cochrane Center, The Cochrane Collaboration, 2011) by pooling the calculated ORs based on the incidence of CI-AKI in the two groups and the reported adjusted ORs from individual studies. Heterogeneity between studies was analyzed by the $I^{2}$ statistic. An $I^{2}$ less than $50 \%$ indicated that the magnitude of heterogeneity may not be important. To explore sources of heterogeneity, metaregression in incidence of CI-AKI was performed using the restricted maximum likelihood method by Stata version 12.0 (Stata Corporation, College Station, TX, USA). Definition of elderly ( $\geq 65$ years or $\geq 75$ years), definition of CI-AKI (serum creatinine increase $\geq 25 \%$ or $0.5 \mathrm{mg} / \mathrm{dL}$, or others), and route of CM administration (intravenous or intra-arterial) were used as covariates in the regression model. Sensitivity analysis was performed by sequentially excluding a single study. $P$-values less than 0.05 in two-tailed tests were considered to be statistically significant.

\section{Results}

We retrieved 159 unique articles. Among these, 32 were excluded based on the abstract alone. The remaining 127 articles included a full-text review, and 105 were excluded for the reasons listed in Figure 1. Consequently, 22 studies ( 21 cohort studies and one randomized controlled trial) with 186,455 patients were identified using our search criteria.

The routes of CM administration were coronary arteries for coronary angiography or intervention in 14 studies; ;,6-18 periphery arteries other than the coronary artery in two studies; $;^{19,20}$ veins for enhanced computed tomography in five studies; ${ }^{21-25}$ and both periphery arteries and veins in one study. ${ }^{26}$ The main characteristics of the study design and participants in the included studies are listed in Table 1.

Among the included 67,831 participants $\geq 65$ years of age, the overall incidence of CI-AKI was $13.6 \%$

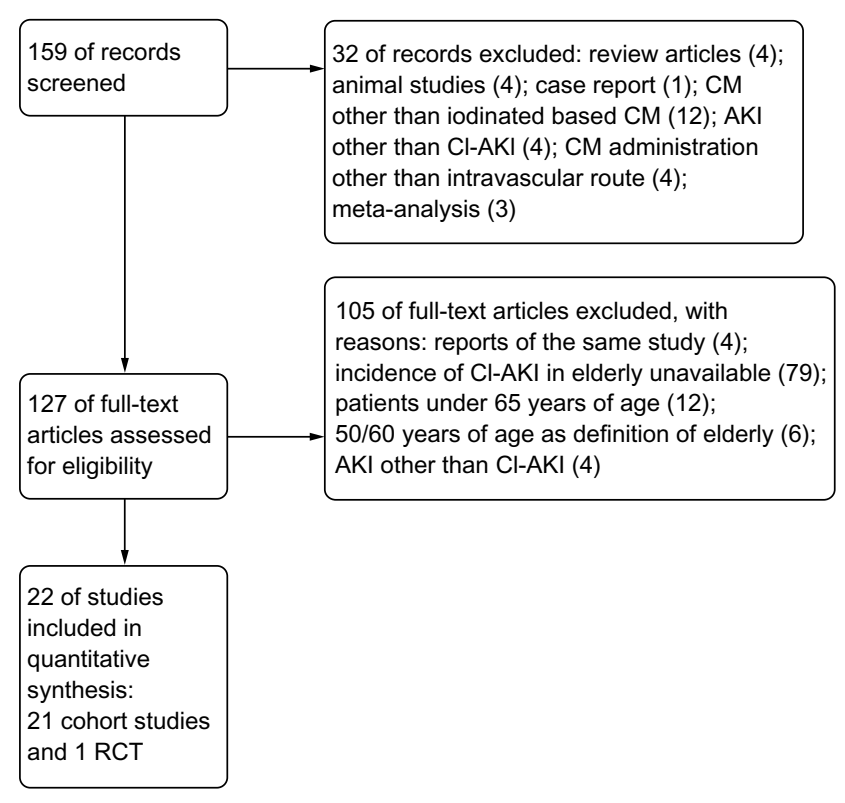

Figure I Search flow diagram.

Abbreviations: AKI, acute kidney injury; $\mathrm{Cl}-\mathrm{AKI}$, contrast-induced acute kidney injury; CM, contrast medium; RCT, randomized controlled trial. 


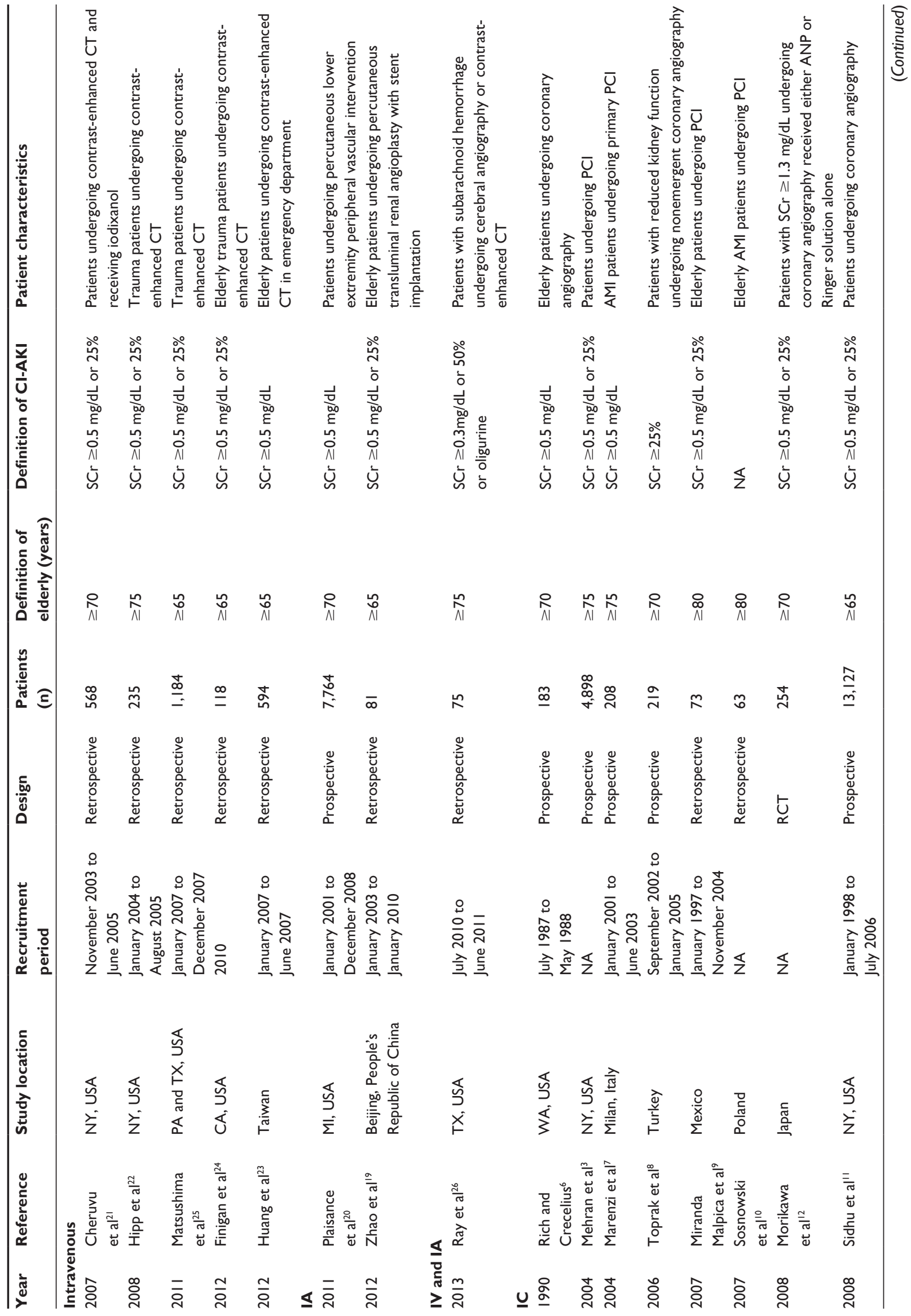




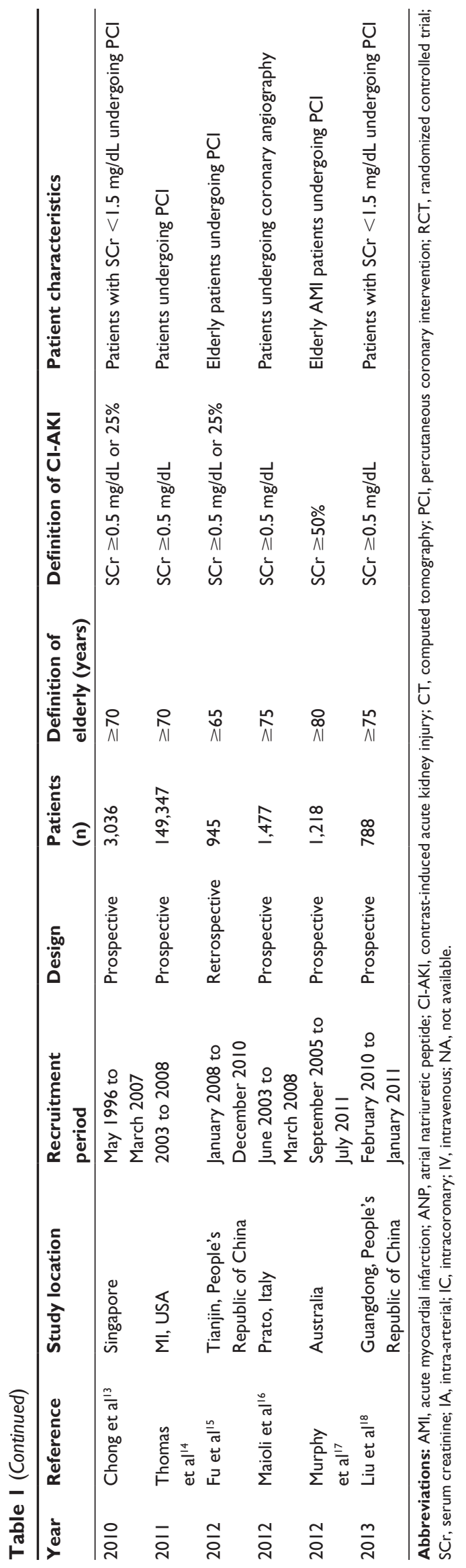

(95\% CI 10.1-18.2, $I^{2}=0.496$, Figure 2). The incidence of CI-AKI in six prespecified subgroups is listed in Table 2. In 12 studies for which the incidence of CI-AKI in both the elderly and younger groups was reported, the pooled OR of CI-AKI in the elderly was 2.10 (95\% CI $1.77-2.48, I^{2}=0.77$, Figure $3 \mathrm{~A}$ ). The risk of CI-AKI in the elderly was consistent across the subsets of the different $\mathrm{CM}$ administration routes. In six studies for which adjusted ORs of CI-AKI in the elderly were reported, the pooled OR of CI-AKI in the elderly was 2.55 (95\% CI 1.85-3.52, $I^{2}=0.34$, Figure 3B).

The metaregression showed that the regression model explained $65.33 \%$ of total between-study variance in incidence of CI-AKI. Definition of elderly was associated with and explained a statistically significant degree of variability $(P=0.002)$. The metaregression model is presented in Table 3. The sensitivity analysis suggested that no single study strongly influenced the overall results, because sequentially excluding one individual study at a time did not affect the movement of the point estimate outside the $95 \%$ CI (data not shown).

\section{Discussion}

In the present study, we report the results of a meta-analysis that pooled the incidence and ORs of CI-AKI in the elderly, categorized into different subsets. To the best of our knowledge, this is the first meta-analysis on this issue.

CI-AKI is an important potential complication following CM-based procedures, including noninvasive enhanced computed tomography or invasive angiography. CI-AKI generally resolves spontaneously in most instances, but patients with CI-AKI tend to experience prolonged hospital stays, increased risk of in-hospital death, and long-term adverse cardiac and renal events. ${ }^{27-30}$ Except for continued volume expansion and minimized CM volume, no pharmacologic prophylaxes have been shown to offer benefit in CI-AKI prevention. ${ }^{31}$ Iodixanol, a new iso-osmolar CM, is not associated with less CI-AKI when compared with low-osmolar CM. ${ }^{32,33}$ Prophylactic hemodialysis as an adjunct to angiography has been shown to be harmful. ${ }^{34}$ Therefore, current practice guidelines for CI-AKI management emphasize risk factor assessment and balancing the relative benefits and risks before any CM-based procedure is performed. ${ }^{31,34,35}$

Advanced age has been recognized for years as an important independent risk factor for the development of CI-AKI. ${ }^{3,6}$ As the population ages, the number of elderly patients referred for CM-based procedures is increasing steadily. ${ }^{14,20}$ On the other hand, limited data regarding the true 


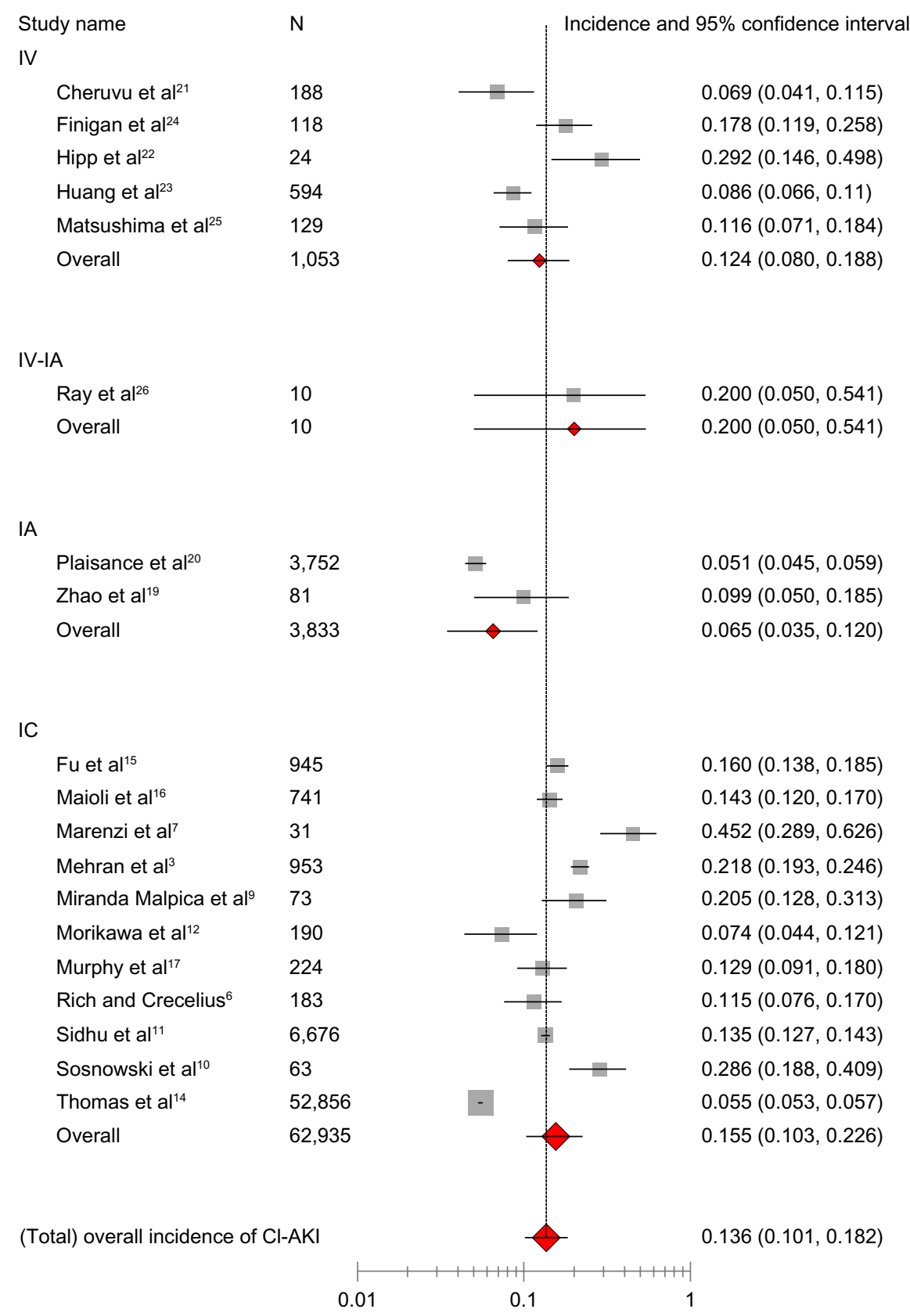

Figure 2 Overall incidence of $\mathrm{Cl}$-AKI in elderly patients (age $\geq 65$ years). Meta-analysis of administration route stratified by pooling the reported incidences of $\mathrm{Cl}$-AKI from individual studies.

Abbreviations: CI-AKI, contrast-induced acute kidney injury; IA, intra-arterial; IC, intracoronary; IV, intravenous; n, number of participants.

Table 2 Incidence of $\mathrm{Cl}-\mathrm{AKI}$ in prespecified subgroups

\begin{tabular}{|c|c|c|c|c|c|}
\hline Subgroup & Studies (n) & Patients (n) & Estimated incidence (\%) & $95 \% \mathrm{Cl}$ & $I^{2}$ statistic \\
\hline \multicolumn{6}{|l|}{ Age (years) } \\
\hline$\geq 75$ & 12 & 23,007 & 16.5 & II.7-22.7 & 0.494 \\
\hline$\geq 80$ & 6 & 20,778 & 13.5 & $8.6-20.6$ & 0.495 \\
\hline \multicolumn{6}{|l|}{$\mathrm{CM}$ administration routes } \\
\hline IC & 11 & 62,935 & 15.5 & $10.3-22.6$ & 0.498 \\
\hline IA, other than via coronary artery & 2 & 3,833 & 6.5 & $3.5-12.0$ & 0.416 \\
\hline IV & 5 & $\mathrm{I}, 053$ & 12.4 & $8.0-18.8$ & 0.442 \\
\hline Other than via coronary artery & 8 & 4,896 & $\mathrm{II}$ & $7.1-16.5$ & 0.471 \\
\hline
\end{tabular}

Abbreviations: Cl-AKI, contrast-induced acute kidney injury; Cl, confidence interval; CM, contrast medium; IA, intra-arterial; IC, intracoronary; IV, intravenous. 
A

$\begin{array}{llc}\text { Elder group } & \text { Younger group } & \begin{array}{c}\text { Odds ratio } \\ \text { inverse variance model, }\end{array} \text { inverse variance model, }\end{array}$ Study Definition of elderly Events Total Events Total Weight random, $95 \% \mathrm{Cl}$ random, $95 \% \mathrm{Cl}$

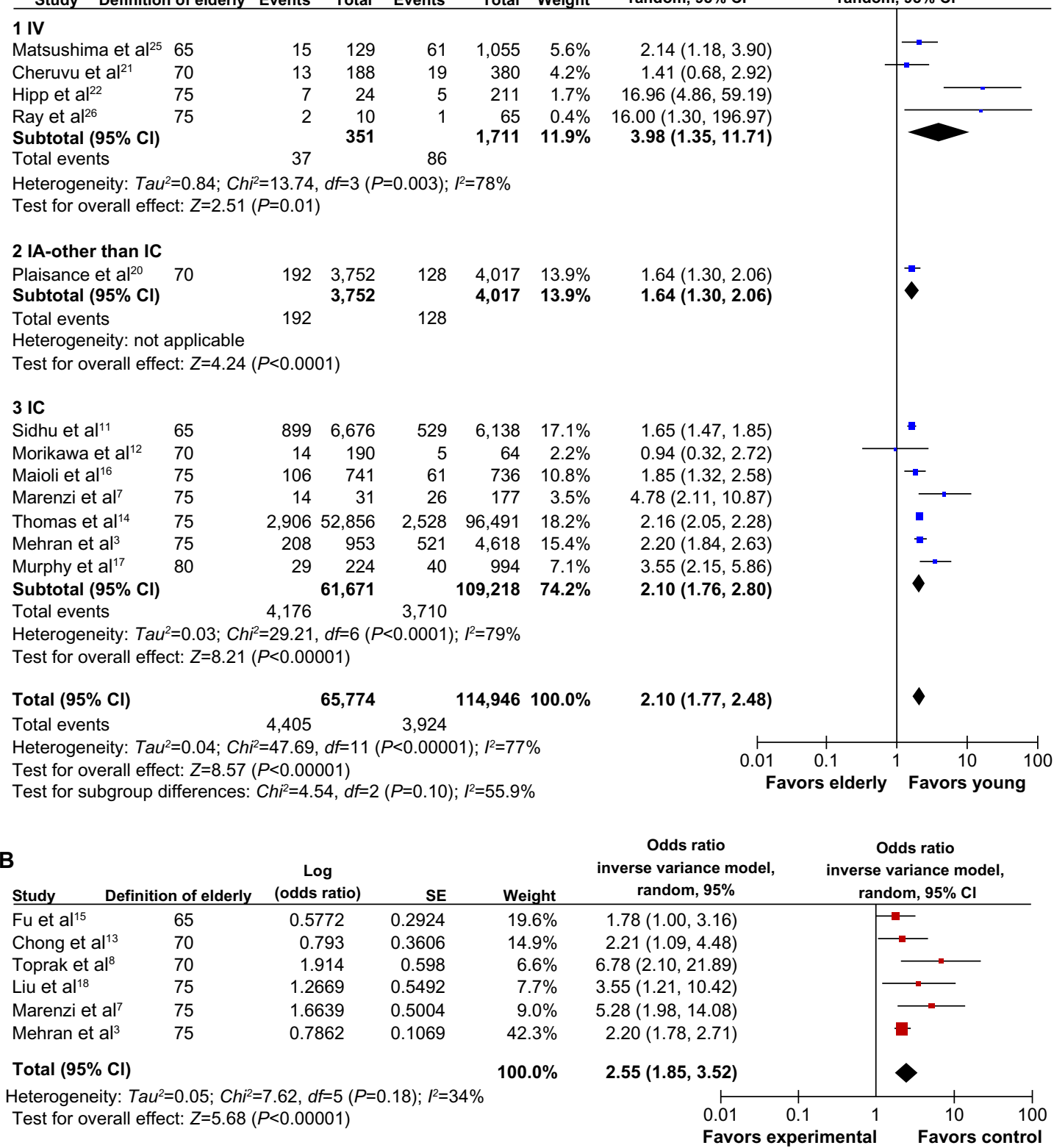

Figure 3 Odds ratios of $\mathrm{Cl}-\mathrm{AKI}$ in the elderly. (A) Meta-analysis of administration route stratified by pooling the calculated odds ratios based on the incidence of $\mathrm{Cl}$-AKI in the elderly and younger groups. (B) Meta-analysis by pooling the reported adjusted odds ratios from individual studies.

Abbreviations: AKI, acute kidney injury; Cl-AKI, contrast-induced acute kidney injury; CM, contrast medium; RCT, randomized controlled trial; Chi, Chi-square test; $\mathrm{Cl}$, confidence interval; df, degrees of freedom; IA, intra-arterial; IC, intracoronary; IV, intravenous; SD, standard deviation; SE, standard error.

Table 3 Metaregression model in incidence of Cl-AKI

\begin{tabular}{llll}
\hline Covariates & $\mathbf{B}^{\mathrm{a}}(\mathbf{9 5} \% \mathrm{CI})$ & $\boldsymbol{P}$-values & Adjusted $\boldsymbol{R}^{\mathbf{2}, \mathrm{b}}$ \\
\hline $\begin{array}{l}\text { Definition } \\
\text { of elderly }\end{array}$ & $0.107(0.047-0.168)$ & 0.002 & $65.33 \%$ \\
$\begin{array}{l}\text { Definition } \\
\text { of Cl-AKI }\end{array}$ & $-0.042(-0.096-0.0 \mathrm{II})$ & 0.109 & \\
$\begin{array}{l}\text { Route of CM } \\
\text { administration }\end{array}$ & $-0.006(-0.070-0.058)$ & 0.844 & \\
\hline
\end{tabular}

Notes: ${ }^{a}$ Coefficient of covariation; ${ }^{b}$ reflects proportion of variability in betweenstudy variance explained by the metaregression model.

Abbreviations: $\mathrm{Cl}-\mathrm{AKI}$, contrast-induced acute kidney injury; $\mathrm{Cl}$, confidence interval; CM, contrast medium. incidence and risk of developing CI-AKI in the elderly affect decision-making. Based on our meta-analysis, we found that: the risk of developing CI-AKI in the elderly is over two times higher than in younger patients, even after adjustment for other risk factors; the estimated overall incidence of CI-AKI following intravascular CM administration is $13.6 \%$ in the elderly, which is higher than the previously reported incidence in an unselected population; ${ }^{2}$ and the trend of a high incidence of CI-AKI in the elderly is consistent across different administration route subgroups. Thus, advanced age 
should be given serious consideration when referring elderly patients for any CM-based procedure.

\section{Limitations}

First, the prevalence of other known risk factors of CI-AKI, such as chronic kidney disease, diabetes, dehydration, and concurrent nephrotoxic medication is high in the elderly. These comorbidities might also play important roles in the development of CI-AKI in the elderly. ${ }^{36} \mathrm{~A}$ metaregression with these risk factors as covariates would investigate the extent of these comorbidities contributing to the onset of CI-AKI in the elderly. However, due to incomplete information of these factors from the original studies, we could not add these factors into our regression model. On the other hand, we had pooled the ORs adjusted by risk factors in our meta-analysis, which might help us to evaluate the sole role of advanced age in the development of CI-AKI. Second, definitions of elderly and CI-AKI varied among the included studies, which brought heterogeneity into our meta-analysis. Metaregression indicated that different definitions of elderly could partially explain the heterogeneity. The age-stratified subgroup analysis we performed would be helpful to reduce the heterogeneity. Although definition of CI-AKI was not shown to be associated with a significant degree of variability by metaregression, the incidence of CI-AKI could have been underestimated in studies using only the absolute increase in serum creatinine as the definition of CI-AKI. Meanwhile, the recently published KDIGO (Kidney Disease: Improving Global Outcomes) guidelines recommended the definition of CI-AKI should be based on both urinary output and changes in serum creatinine, and the severity of CI-AKI should be graded the same as the definition of acute kidney injury. ${ }^{34}$ This criteria was not used in our meta-analysis because the vast majority of clinical trials on CI-AKI used the definition based on serum creatinine alone and without grading. Third, we used just PubMed as a search engine. Although we identified 186,455 patients from 22 studies for the present study, using other search engines might yield more information on the incidence and risk of developing CI-AKI following intravascular contrast administration in elderly patients. Finally, no data regarding the impact of CI-AKI on a patient's clinical course and prognosis, and no conclusive management strategy for the elderly are available. Further studies are needed to address these issues.

\section{Conclusion}

Elderly patients are at greater risk for the development of CIAKI. The overall incidence of CI-AKI in patients $\geq 65$ years of age is up to $13.6 \%$.

\section{Acknowledgments}

We especially thank Dr Jinjin Zhang for his assistance with performing the metaregression and help with revising the Materials and methods section. This work is supported by the Program for Outstanding Medical Academic Leader from Shanghai Municipal Health Bureau.

\section{Author contributions}

WS and BH developed the study protocol; WS and TZ performed the literature search; WS, TZ, and JP analyzed the data; WS, LS, and BH interpreted data and prepared the manuscript; and WS, TZ, JP, LS and BH revised the manuscript.

\section{Disclosure}

The authors report no conflicts of interest in this work.

\section{References}

1. Seeliger E, Sendeski M, Rihal CS, Persson PB. Contrast-induced kidney injury: mechanisms, risk factors, and prevention. Eur Heart J. 2012;33:2007-2015.

2. McCullough PA. Contrast-induced acute kidney injury. J Am Coll Cardiol. 2008;51:1419-1428.

3. Mehran R, Aymong ED, Nikolsky E, et al. A simple risk score for prediction of contrast-induced nephropathy after percutaneous coronary intervention: development and initial validation. J Am Coll Cardiol. 2004;44:1393-1399.

4. Zhang T, Shen L, Hu L, He B. Optimal duration of dual-antiplatelet therapy following drug-eluting stent implantation: a meta-analysis. J Clin Pharmacol. 2013;53:345-351.

5. Wallace BC, Schmid CH, Lau J, Trikalinos TA. Meta-Analyst: software for meta-analysis of binary, continuous and diagnostic data. BMC Med Res Methodol. 2009;9:80.

6. Rich MW, Crecelius CA. Incidence, risk factors, and clinical course of acute renal insufficiency after cardiac catheterization in patients 70 years of age or older. A prospective study. Arch Intern Med. 1990;150:1237-1242.

7. Marenzi G, Lauri G, Assanelli E, et al. Contrast-induced nephropathy in patients undergoing primary angioplasty for acute myocardial infarction. J Am Coll Cardiol. 2004;44:1780-1785.

8. Toprak O, Cirit M, Yesil M, et al. Metabolic syndrome as a risk factor for contrast-induced nephropathy in non-diabetic elderly patients with renal impairment. Kidney Blood Press Res. 2006;29:2-9.

9. Miranda Malpica E, Pena Duque MA, Castellanos J, et al. Predictors of mortality and adverse outcome in elderly high-risk patients undergoing percutaneous coronary intervention. Arch Cardiol Mex. 2007;77:194-199.

10. Sosnowski C, Janeczko-Sosnowska E, Wozniak J, et al. Primary coronary intervention in diabetic octogenarians with acute ST elevation myocardial infarction. Kardiol Pol. 2007;65:1181-1186.

11. Sidhu RB, Brown JR, Robb JF, et al. Interaction of gender and age on post cardiac catheterization contrast-induced acute kidney injury. Am J Cardiol. 2008;102:1482-1486.

12. Morikawa S, Sone T, Tsuboi H, et al. Renal protective effects and the prevention of contrast-induced nephropathy by atrial natriuretic peptide. J Am Coll Cardiol. 2009;53:1040-1046.

13. Chong E, Poh KK, Liang S, Tan HC. Risk factors and clinical outcomes for contrast-induced nephropathy after percutaneous coronary intervention in patients with normal serum creatinine. Ann Acad Med Singapore. 2010;39:374-380.

14. Thomas MP, Moscucci M, Smith DE, et al. Outcome of contemporary percutaneous coronary intervention in the elderly and the very elderly: insights from the Blue Cross Blue Shield of Michigan Cardiovascular Consortium. Clin Cardiol. 2011;34:549-554. 
15. Fu N, Li X, Yang S, et al. Risk score for the prediction of contrastinduced nephropathy in elderly patients undergoing percutaneous coronary intervention. Angiology. 2012;64:188-194.

16. Maioli M, Toso A, Leoncini M, Gallopin M, Musilli N, Bellandi F. Persistent renal damage after contrast-induced acute kidney injury: incidence, evolution, risk factors, and prognosis. Circulation. 2012;125: 3099-3107.

17. Murphy JC, Kozor RA, Figtree G, et al. Procedural and in-patient outcomes in patients aged 80 years or older undergoing contemporary primary percutaneous coronary intervention. EuroIntervention. 2012;8: 912-919.

18. Liu Y, Tan N, Chen J, et al. The relationship between hyperuricemia and the risk of contrast-induced acute kidney injury after percutaneous coronary intervention in patients with relatively normal serum creatinine. Clinics (Sao Paulo). 2013;68:19-25.

19. Zhao J, Cheng Q, Zhang X, Li M, Liu S, Wang X. Efficacy of percutaneous transluminal renal angioplasty with stent in elderly male patients with atherosclerotic renal artery stenosis. Clin Interv Aging. 2012;7: $417-422$.

20. Plaisance BR, Munir K, Share DA, et al. Safety of contemporary percutaneous peripheral arterial interventions in the elderly insights from the BMC2 PVI (Blue Cross Blue Shield of Michigan Cardiovascular Consortium Peripheral Vascular Intervention) registry. JACC Cardiovasc Interv. 2011;4:694-701.

21. Cheruvu B, Henning K, Mulligan J, et al. Iodixanol: risk of subsequent contrast nephropathy in cancer patients with underlying renal insufficiency undergoing diagnostic computed tomography examinations. J Comput Assist Tomogr. 2007;31:493-498.

22. Hipp A, Desai S, Lopez C, Sinert R. The incidence of contrast-induced nephropathy in trauma patients. Eur J Emerg Med. 2008;15:134-139.

23. Huang MK, Hsu TF, Chiu YH, et al. Risk factors for acute kidney injury in the elderly undergoing contrast-enhanced computed tomography in the emergency department. J Chin Med Assoc. 2012;76:271-276.

24. Finigan R, Pham J, Mendoza R, et al. Risk for contrast-induced nephropathy in elderly trauma patients. Am Surg. 2012;78:1114-1117.

25. Matsushima K, Peng M, Schaefer EW, Pruitt JH, Kashuk JL, Frankel HL. Posttraumatic contrast-induced acute kidney injury: minimal consequences or significant threat? J Trauma. 2011;70:415-419.

26. Ray B, Rickert KL, Welch BG, et al. Development of contrast-induced nephropathy in subarachnoid hemorrhage: a single center perspective. Neurocrit Care. 2013;19(2):150-156.
27. James MT, Samuel SM, Manning MA, et al. Contrast-induced acute kidney injury and risk of adverse clinical outcomes after coronary angiography: a systematic review and meta-analysis. Circ Cardiovasc Interv. 2013;6:37-43.

28. Klima T, Christ A, Marana I, et al. Sodium chloride vs sodium bicarbonate for the prevention of contrast medium-induced nephropathy: a randomized controlled trial. Eur Heart J. 2012;33:2071-2079.

29. Rihal CS, Textor SC, Grill DE, et al. Incidence and prognostic importance of acute renal failure after percutaneous coronary intervention. Circulation. 2002;105:2259-2264.

30. Markota D, Markota I, Starcevic B, Tomic M, Prskalo Z, Brizic I. Prevention of contrast-induced nephropathy with $\mathrm{Na} / \mathrm{K}$ citrate. Eur Heart J. 2013;34:2362-2367.

31. Stacul F, van der Molen AJ, Reimer P, et al. Contrast induced nephropathy: updated ESUR Contrast Media Safety Committee guidelines. Eur Radiol. 2011;21:2527-2541.

32. Reed M, Meier P, Tamhane UU, Welch KB, Moscucci M, Gurm HS. The relative renal safety of iodixanol compared with low-osmolar contrast media: a meta-analysis of randomized controlled trials. JACC Cardiovasc Interv. 2009;2:645-654.

33. From AM, Al Badarin FJ, McDonald FS, Bartholmai BJ, Cha SS, Rihal CS. Iodixanol versus low-osmolar contrast media for prevention of contrast induced nephropathy: meta-analysis of randomized, controlled trials. Circ Cardiovasc Interv. 2010;3:351-358.

34. Fliser D, Laville M, Covic A, et al. A European Renal Best Practice (ERBP) position statement on the Kidney Disease Improving Global Outcomes (KDIGO) clinical practice guidelines on acute kidney injury: part 1: definitions, conservative management and contrast-induced nephropathy. Nephrol Dial Transplant. 2012;27:4263-4272.

35. Levine GN, Bates ER, Blankenship JC, et al. 2011 ACCF/AHA/SCAI Guideline for Percutaneous Coronary Intervention. A report of the American College of Cardiology Foundation/American Heart Association Task Force on Practice Guidelines and the Society for Cardiovascular Angiography and Interventions. J Am Coll Cardiol. 2011;58:E44-E122.

36. Toprak O. Risk markers for contrast-induced nephropathy. Am J Med Sci. 2007;334:283-290. 


\section{Supplementary material Search strategy in MEDLINE (from 1966 to June 20I3)}

For the theme of "CI-AKI": "contrast induced nephropathy" [All Fields] OR "contrast associated nephropathy" [All Fields] OR "radiocontrast induced nephropathy" [All Fields] OR "contrast nephropathy" [All Fields] OR "contrast nephrotoxicity" [All Fields] OR "contrast induced nephrotoxicity" [All Fields] OR "contrast associated nephrotoxicity" [All Fields] OR "contrast induced acute renal failure" [All Fields] OR "contrast induced acute kidney injury" [All Fields].

For the theme of "Elderly": "Aged" [Majr] OR "Aged, 80 and over" [MESH] OR "Frail Elderly" [MESH] OR "aged" [Title/Abstract] OR "age group" [Title/Abstract] OR "age groups" [Title/Abstract] OR "elderly" [Title/Abstract] OR "elder population" [Title/Abstract] OR "geriatric" [Title/Abstract] OR "senior" [Title/Abstract] OR "seniors"
[Title/Abstract] OR “old persons" [Title/Abstract] OR "older adult" [Title/Abstract] OR "older adults" [Title/Abstract] OR "older patient" [Title/Abstract] OR "older patients" [Title/ Abstract] OR "65 years" [All Fields] OR "75 years" [All Fields] OR "80 years" [All Fields].

For the theme of "clinical study": (randomized controlled trial[pt]) OR (controlled clinical trial[pt]) OR (randomized[tiab]) OR (placebo[tiab]) OR (drug therapy[sh]) OR (randomly[tiab]) OR (trial[tiab]) OR (groups[tiab]) OR “Clinical Trial” [Publication Type] OR "Comparative Study" [Publication Type] OR "Clinical Trials as Topic" [MESH] OR "Epidemiologic Studies" [MESH] OR "Registries" [MESH] OR longitud*[tiab] OR prospectiv*[tiab] OR retrospectiv*[tiab] OR observation*[tiab] OR cohort*[tiab] OR registry[tiab] OR "clinical trial” [tiab] OR "case control" [tiab] OR "cross section" [tiab]) NOT (animals[mh] NOT humans[mh]).

Three comprehensive search themes were combined by the Boolean operator "AND."
Clinical Interventions in Aging

\section{Publish your work in this journal}

Clinical Interventions in Aging is an international, peer-reviewed journal focusing on evidence-based reports on the value or lack thereof of treatments intended to prevent or delay the onset of maladaptive correlates of aging in human beings. This journal is indexed on PubMed Central, MedLine, the American Chemical Society's 'Chemical Abstracts Ser-

\section{Dovepress}

vice' (CAS), Scopus and the Elsevier Bibliographic databases. The manuscript management system is completely online and includes a very quick and fair peer-review system, which is all easy to use. Visit $\mathrm{http}: / / \mathrm{ww}$.dovepress.com/testimonials.php to read real quotes from published authors. 